\title{
Decreased Time To Peak Drug Blood Concentration
}

National Cancer Institute

\section{Source}

National Cancer Institute. Decreased Time To Peak Drug Blood Concentration. NCI

Thesaurus. Code C54608.

Decreased time following drug administration at which the peak drug blood concentration occurs. 